\title{
Waste Electrical and Electronic Equipment Processing as Thermoplastic Composites ${ }^{\dagger}$
}

\author{
Elena Ramona Andrei 1,*, Andreea Gabriela Oporan ${ }^{1}$, Paul Ghioca ${ }^{1}$, Lorena Iancu 1,2, \\ Madalina David ${ }^{1,2}$, Rodica-Mariana Ion ${ }^{1,2}$, Zina Vuluga ${ }^{1}$, Bogdan Spurcaciu ${ }^{1}$ and \\ Ramona Marina Grigorescu ${ }^{1}$ \\ 1 National Institute for Research \& Development in Chemistry \& Petrochemistry-ICECHIM, \\ 202 Splaiul Independentei, 060021 Bucharest, Romania; andreea.oporan@icechim.ro (A.G.O.); \\ paul.ghioca@icechim.ro (P.G.); lorena.iancu@icechim.ro (L.I.); madalina.david@icechim.ro (M.D.); \\ rodica.ion@icechim.ro (R.M.-I.); zvuluga@icechim.ro (Z.V.); bogdan.spurcaciu@icechim.ro (B.S.); \\ ramona.grigorescu@icechim.ro (R.M.G.) \\ 2 Doctoral School of Materials Engineering Department, Valahia University, 13 Aleea Sinaia, \\ 130004 Targoviste, Romania \\ * Correspondence: ramona.andrei@icechim.ro \\ † Presented at the 16th International Symposium "Priorities of Chemistry for a Sustainable Development" \\ PRIOCHEM, Bucharest, Romania, 28-30 October 2020.
}

Published: 12 November 2020

Keywords: melt processing; extrusion molding; injection molding; waste electrical and electronic equipment

In the last decades, the waste electric and electronic equipment (WEEE) has increased substantially due to an accelerated development of the economy and the lifetime of electronics. Intensive research has been focused on reusing the waste in order to decrease the amount of incinerated or landfill waste. The aim of the paper was to melt process some compositions based on the polystyrene fraction of WEEE and on the non-metallic fraction of waste printed circuit boards (WPCB), and compositions that were selected considering the correlation between the mechanical properties of the polymeric composites and the economic advantages arising from the use of a larger amount of wastes [1-3].

For the WEEE composite, the majority component was the polystyrene fraction of the waste, consisting of polystyrene (PS), acrylonitrile-butadiene-styrene (ABS), and high-impact polystyrene (HIPS). The WPCB composite contained recycled polypropylene (rPP) and the non-metallic waste. For both compounds, a styrene-butadiene block copolymer (SBS) and a maleinized and hydrogenated block polymer (SEBS-MA) were used as modifiers. The composites were processed by extrusion and injection molding, and specimens with a uniform aspect and reproducible characteristics were obtained (Figure 1). Tensile and impact properties of the resulted composite materials were determined.

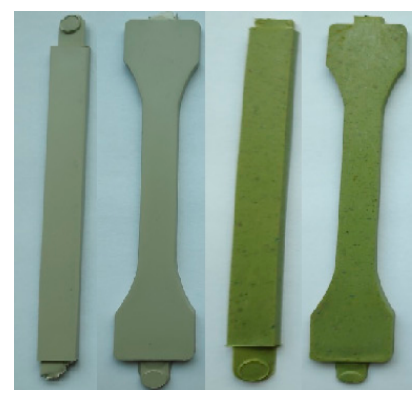

Figure 1. Uniform aspect of the injected items. 
For the studied compounds, it could be observed that some defects appeared at extrusion, such as interior voids, extrudate swelling, shark skin fractures, and variable flow of the polymeric melt. Defect-free extrudates can be granulated and used for injection molding by modifying the processing conditions. The composition influence on mechanical and impact properties of waste composites highlighted that the obtained impact strength, tensile strength, and elongation at break were comparable with the characteristics of commercial polymers used in the industry.

The processability of the waste composites was verified by obtaining extruded and injected items in suitable conditions. Their aspect was homogeneous, without surface defects and with high mechanical properties.

Acknowledgments: This paper was supported by a grant of the Romanian Ministry of Research and Innovation, CCCDI-UEFISCDI, contract number 84 PCCDI/2018, within PNCDI III and by project no. PN.19.23.03.01, contract no. 23N/2019 within the Nuclear Program.

\section{References}

1. Goodship, V.; Stevels, A. Waste Electrical and Electronic Equipment (WEEE) Handbook; Woodhead Publishing: Cambridge, UK, 2012; pp. 1-724.

2. Grigorescu, R.M.; Ghioca, P.; Iancu, L.; Grigore, M.E.; Ion, R.M.; Nicolae, C.A.; Gabor, R.; Filipescu, M.I.; Rapa, M.; Trusca, R.D. Impact strength elastomer composites based on polystyrene components separated from waste electrical and electronic equipment. J. Appl. Polym. Sci. 2020, 137, 48329.

3. Grigorescu, R.M.; Ghioca, P.; Iancu, L.; David, M.E.; Andrei, E.R.; Filipescu, M.I.; Ion, R.-M.; Vuluga, Z.; Anghel, I.; Sofran, I.-E. Development of thermoplastic composites based on recycled polypropylene and waste printed circuit boards. Waste Manag. 2020, 118, 391-401.

Publisher's Note: MDPI stays neutral with regard to jurisdictional claims in published maps and institutional affiliations.

(C) 2020 by the authors. Licensee MDPI, Basel, Switzerland. This article is an open access article distributed under the terms and conditions of the Creative Commons Attribution (CC BY) license (http://creativecommons.org/licenses/by/4.0/). 